www.jmscr.igmpublication.org

Impact Factor 5.244

Index Copernicus Value: 83.27

ISSN (e)-2347-176x ISSN (p) 2455-0450

crossref DOI: _https://dx.doi.org/10.18535/jmscr/v4i10.113

Journal Of Medical Science And Clinical Research

\title{
Reversible Night Blindness in an Adult - A Case Report
}

\author{
Authors \\ Tharini $\mathbf{S}^{\mathbf{1}}$, Naresh Babu ${ }^{2}$ \\ ${ }^{1}$ Resident, Aravind Eye Institute, Madurai \\ ${ }^{2}$ Retina consultant and Surgeon, Aravind Eye Institute, Madurai \\ Corresponding Author \\ Dr S.Tharini \\ Email:drtharini88@gmail.com
}

\begin{abstract}
Vitamin A deficiency is rare in adults, still rarer is the fundus changes due to it. We report a case of 55 years old male with post gastric bypass surgery status, presenting with defective distant vision for 2 years whom on investigation revealed fundus changes, defective colour vision, abnormalities in OCT and ERG and low serum retinol levels who was treated successfully with Vitamin A therapy.
\end{abstract}

KEY WORDS: Fundus, Vitamin A

\section{INTRODUCTION}

Vitamin A deficiency due to malnutrition is the leading cause of childhood blindness in the developing world. It is rare in adults and can occur as a result of malabsorption, poor vitamin metabolism due to liver disease or cystic fibrosis. Recently, in developing countries like India, due to increased number of bariatric procedures and malabsorption syndromes, vitamin A deficiency in adults is on the rise. We report two cases with reversible night blindness due to vitamin A deficiency.

\section{CASE REPORT}

A 55 year old male presented with complaints of defective distant vision in both eyes for 2 years, which started initially with difficulty in night vision. He was a known diabetic for past 1 year, on insulin therapy. He underwent gastric bypass surgery for peptic ulcer disease 10 years back. On
Examination, his best corrected visual acuity in both the eyes was 5/60 NIG NIP. Anterior segment was normal in both eyes. Fundus examination of both eyes showed mild disc pallor with diffuse retinal pigment epithelial mottling (fig $1 \& 2$ ).

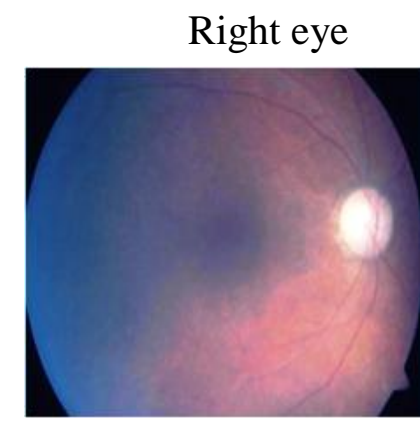

Fig-1

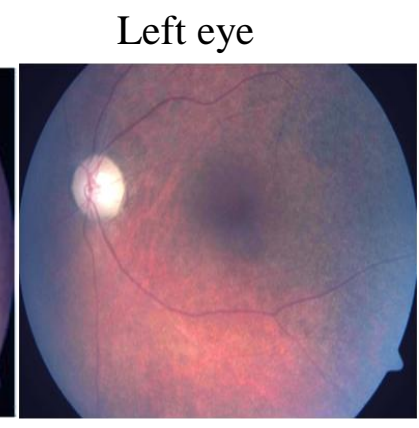

Fig-2
On Colour vision testing he could no tread any plate. Central fields of both his eyes showed tubular vision with peripheral constriction of fields. Optical Coherence Tomography (OCT) showed thin epi-retinal membrane with disruption 
of inner segment-outer segment junction. ElectroRetinogram (ERG) showed diminished rod and cone responses (fig-3)

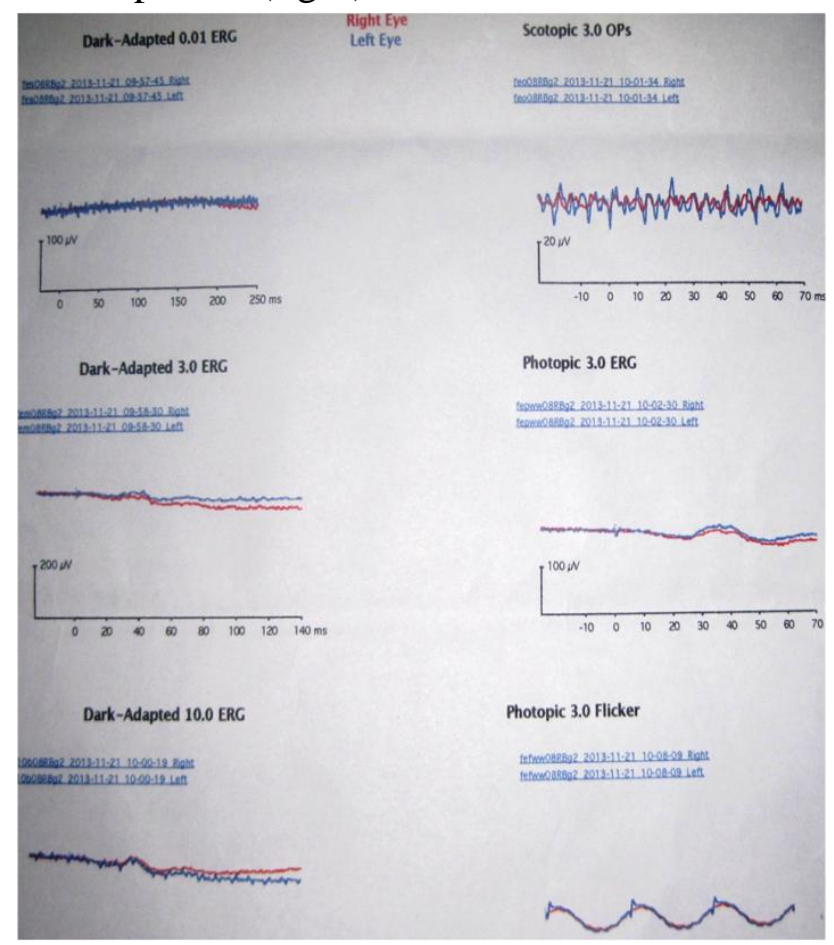

Fig-3: ERG picture before treatmentnt

A differential diagnosis of Albinism, Cataract, Glaucoma, Gyrateatrophy, Oguchi's Disease, Optic atrophy, Retinitis Pigmentosa, Siderosis, Uncorrected myopia, Vitamin A deficiency and Zinc deficiency were considered. After ruling out other possibilities Vitamin A deficiency was strongly considered. His serum retinol level was $<0.1 \mathrm{mg} / \mathrm{l} \quad(\mathrm{N}-0.3-0.6 \mathrm{mg} / \mathrm{l})$. Hence the same diagnosis of was made and he was started on injection Vitamin A5000 IU intramuscularly for 10 days and oral Vitamin A capsules 10,000 IU twice daily for 1 month.After one month of treatment, his vision had returned to normal, visual acuity had improved to $6 / 6$ in both the eyes and fundus examination was normal in both the eyes. He also showed improvement in colour vision. His serum vitamin A level was still below normal but had increased to $0.26 \mathrm{mg} / \mathrm{L}$. A repeat visual field was performed at that visit which showed an improvement in both visual fields. Repeat ERG showed improved rod and cone responses (fig-4 \& 5).

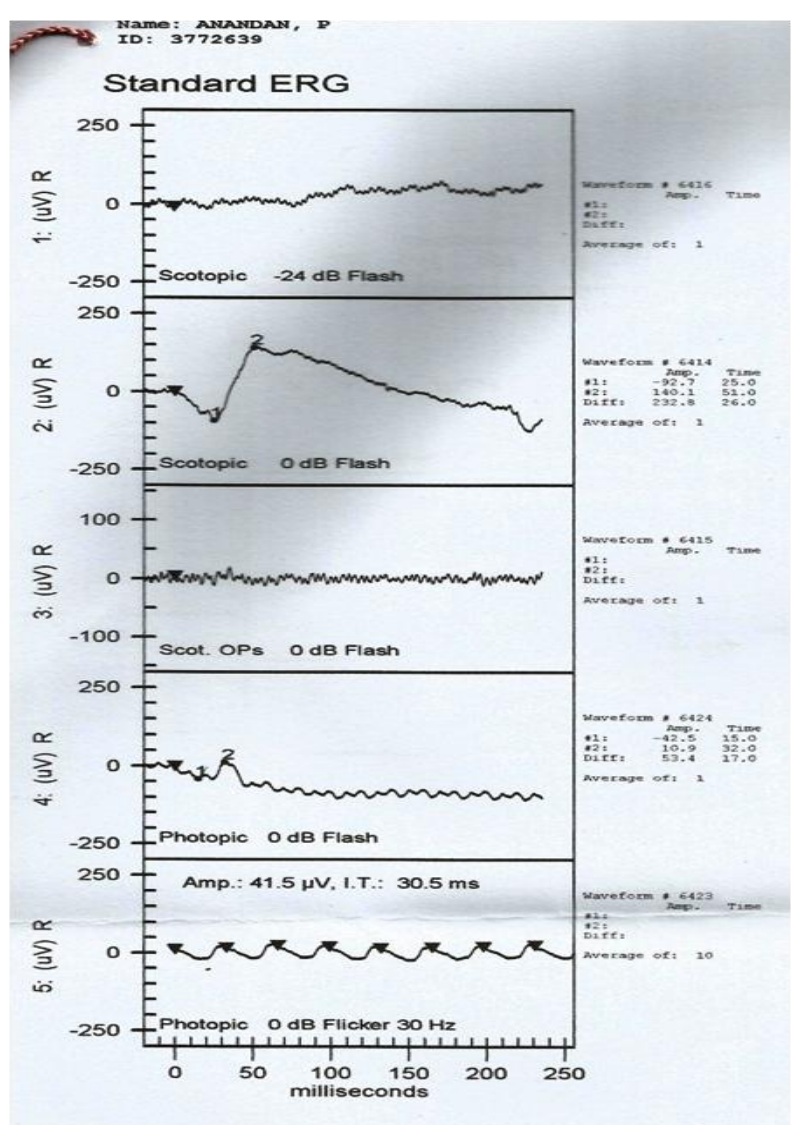

Fig-4

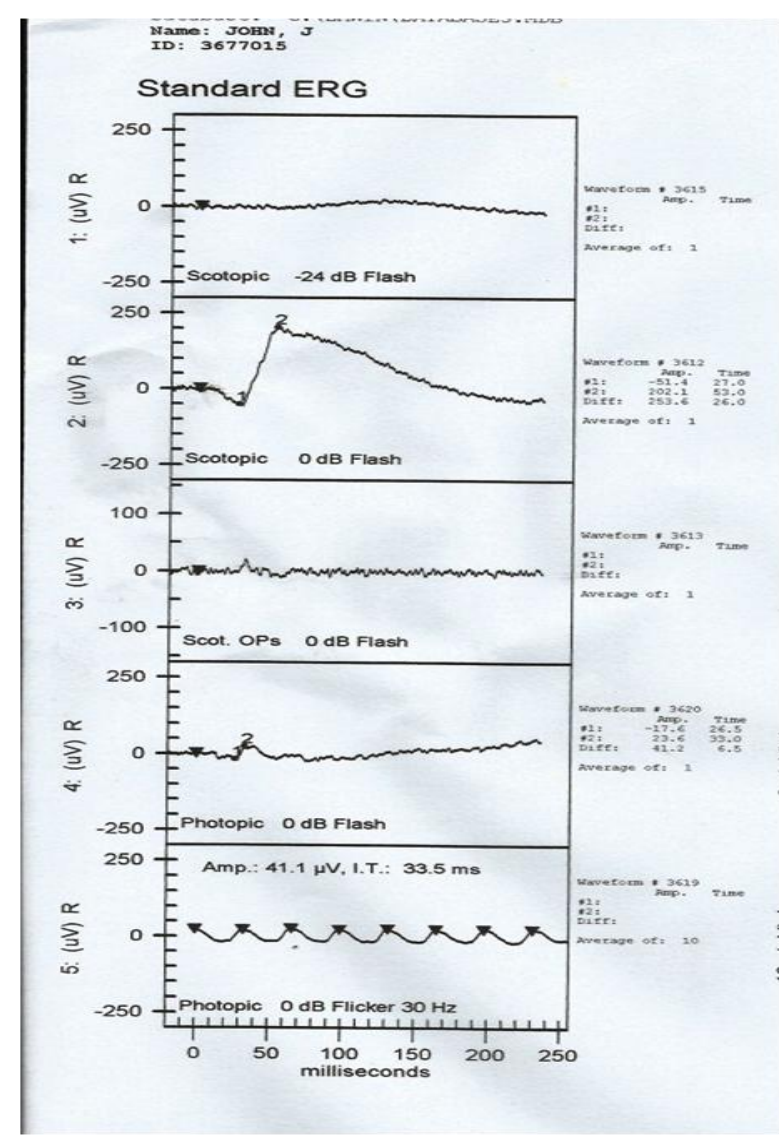

Fig-5 


\section{DISCUSSION}

Xerophthalmia is a term used to describe the spectrum of ocular disease that occurs from vitamin A deficiency. These changes include dry eye (xerosis), corneal ulceration and melting (kerotomalacia), night blindness (nyctalopia), and retinopathy ${ }^{[1]}$ Vitamin $A$ is also essential for immune function, and affected children are more susceptible to severe infections, such as measles. Vitamin A is ingested in the form of retinaldehyde from milk, meat, fish, liver, and eggs. It is also ingested as carotene from green leafy vegetables, yellow fruits, and red palm oil. These compounds are stored in the liver in the form of retinylpamitate. The aldehyde form of vitamin A, retinaldehyde, combines with the protein opsin in the rods to create rhodopsin, which is a photosensitive pigment. A similar process takes place in the cones. During photo transduction, some retinal is lost so a constant supply of vitamin $\mathrm{A}$ is needed.

Vitamin A deficiency can therefore lead to night blindness with associated visual field changes and a depressed ERG. Vitamin A is also necessary for the maintenance of specialized epithelial surfaces. In the conjunctiva, loss of goblet cells and squamous cell metaplasia leads to dryness or xerosis. Bitot's spotsare perilimbalgray plaques of keratinized conjunctival debris overlying an area of $x^{2}{ }^{[5]}$.A full-thickness liquefy active necrosis of the cornea (keratomalacia) can also occur. Finally, retinopathy in the form of yellow or white punctuate dots can be seen in the retinal periphery ${ }^{[4]}$.Testing for vitamin A deficiency is available in the form of retinol, retinylpamitate, and retinol binding protein levels.

The World Health Organization recommends 200,000 IU of vitamin A for 2 days followed by another dose 2 weeks later for severely malnourished children older than 12 years of age with vitamin A deficiency (WHO 1999); [6] however, more recent case studies have shown that much less supplementation can lead to reversal of symptoms. Functional improvement of the retina is likely to occur before ophthalmoscopically evident improvement of structural changes in fundus. ${ }^{[3]}$ Vitamin A can also be given intramuscularly if there is a concern for severe malabsorption or corneal involvement requiring a faster recovery. ${ }^{[2]}$

\section{CONCLUSION}

Vitamin A deficiency in adults is rare and fundus changes in vitamin A deficiency is still rarer .Our patient had a fairly quick recovery on vitamin A supplementation. This reinforces the importance of considering vitamin A supplementation in all patients who have undergone bowel resection and in patients with underlying liver disease.

\section{REFERENCES}

1. Ocular manifestations of vitamin a deficiency-G. Venkataswamy Department Of Ophthalmology, Madurai Medical College, Madurai, India . Brit. J. Ophthal. (1967) $51,854$.

2. Sommer A, Sugana T, Djunaedi E, Green R. Vitamin A responsive panocularxerophthalmia in a healthy adult. Arch Ophthalmol 1978;96:1630-1634

3. Marsha A. Apushkin, MD, Gerald A. Fishman, MD. Improvement in visual function and fundus findings for a patient with vitaminA deficient retinopathy. Retina. The Journal of Retinal and Vitreous diseases 2005 Vol 25 Number

4. Genead M. Fishman G. Lindeman M. Fundus white spots and acquired night blindness due to vitamin A deficiency. Doc Ophthalmol 2009;119:229-233.

5. Lee, W. Hamiton S, Harris J, Schwab I. Ocular Complication of Hypovitaminosis A after Bariatric Surgery. Ophthalmology 2005;112:1031-1034.

6. World Health Organization. Management of severe malnutrition: a manual for physicians and other senior health workers. 1999:17-18 